sma

\title{
Alpha-Synuclein Amyloid Oligomers Act as Multivalent Nanoparticles to Cause Hemifusion in Negatively Charged Vesicles
}

\author{
Anja N. D. Stefanovic, Mireille M. A. E. Claessens, * Christian Blum, \\ and Vinod Subramaniam*
}

Alpha-synuclein $(\alpha \mathrm{S})$ is a brain specific protein found in presynaptic terminals where it colocalizes with synaptic vesicles, ${ }^{[1]}$ suggesting that $\alpha \mathrm{S}$ interacts with membranes. The affinity of $\alpha \mathrm{S}$ for phospholipid bilayers has been confirmed; $\alpha \mathrm{S}$ directly binds to negatively charged vesicles. ${ }^{[2,3]}$ Although the exact function of $\alpha \mathrm{S}$ is still unclear, membrane binding is thought to be critical for its physiological function. ${ }^{[4]} \mathrm{Mem}$ brane-bound $\alpha \mathrm{S}$ has been suggested to play a role in vesicle trafficking, ${ }^{[5]}$ synaptic vesicle fusion, ${ }^{[6]}$ and in the regulation of the mitochondrial fusion/fission machinery. ${ }^{[4]}$ The mechanism by which $\alpha \mathrm{S}$ affects these membrane remodeling processes is still under debate. Binding of $\alpha \mathrm{S}$ might affect fusion and fission by directly changing the physical properties of membranes. Observed $\alpha \mathrm{S}$ binding-induced changes in membrane properties include the adoption of a fusion-prone positive mean curvature and a negative Gaussian curvature in phosphatidylserine (PS):phosphatidylcholine (PC) membranes ${ }^{[7]}$ and the annealing of raft-like membrane structures involved in membrane fusion. ${ }^{[8]}$ Besides affecting membrane properties, the binding of two helical domains of $\alpha \mathrm{S}$ to opposing membranes has been suggested to result in the close contact required for membrane fusion. ${ }^{[9]}$ An alternative hypothesis is that $\alpha \mathrm{S}$ does not directly affect fusion and fission but is part of the SNARE (SNAP (soluble NSF attachment protein)

\author{
Dr. A. N. D. Stefanovic, Prof. M. M. A. E. Claessens, \\ Dr. C. Blum, Prof. V. Subramaniam \\ Nanobiophysics, MESA+ Institute for Nanotechnology \\ Faculty of Science and Technology \\ University of Twente \\ PO Box 217, 7500, AE, Enschede, The Netherlands \\ E-mail:m.m.a.e.claessens@utwente.nl; \\ subramaniam@amolf.nl \\ Prof. M. M. A. E. Claessens, Prof. V. Subramaniam \\ MIRA Institute for Biomedical Technology \\ and Technical Medicine \\ University of Twente \\ PO Box 217, 7500, AE, Enschede, The Netherlands \\ Prof. V. Subramaniam \\ FOM Institute AMOLF \\ Science Park 104 1098, XG, Amsterdam, The Netherlands
}

DOI: 10.1002/smll.201402674 receptor) complex fusion machinery and promotes SNAREmediated fusion. ${ }^{[10]}$

Not only the putative function of $\alpha \mathrm{S}$, but also its role in Parkinson's disease (PD) pathology has been associated with the ability to bind membranes. The toxicity of this aggregation process may not involve cross- $\beta$-sheet rich fibrils; instead intermediate oligomeric structures are thought to be responsible. ${ }^{[11]}$ These oligomers are typically a few nanometers in radius and the aggregation number, that is, the number of monomers forming one oligomer, has been shown to vary with the aggregation conditions. ${ }^{[12-14]}$

The toxicity mechanism proposed for amyloid oligomers is remarkably similar to that proposed for inorganic nanoparticles. ${ }^{[15]}$ Positively charged nanoparticles have a high affinity for negatively charged cellular membranes. ${ }^{[16]}$ The resulting higher cellular uptake of positively charged nanoparticles compared to negatively charged ones is thought to play a role in the disruption of the integrity of cellular membranes. ${ }^{[17]}$ In in vitro tests various nanoparticles have been shown to disrupt supported lipid bilayers by the formation of holes, membrane thinning, and/or membrane erosion. ${ }^{[18]}$ Similar observations have been made for $\alpha \mathrm{S}$ oligomers: $\alpha \mathrm{S}$ oligomer binding results in permeabilization of highly charged model lipid bilayers ${ }^{[19-21]}$ by a mechanism that has been proposed to either involve membrane thinning ${ }^{[22]}$ or the formation of membrane spanning pores. ${ }^{[23]}$ These similarities between inorganic nanoparticles and amyloid oligomers inspired us to think of $\alpha \mathrm{S}$ oligomers as multivalent, biological nanoparticles.

Whether disruption of membrane integrity is indeed the major damage process in vivo remains to be elucidated. In yeast cells, $\alpha \mathrm{S}$ overexpression and the tendency of the protein to misfold and oligomerize interfered with transport pathways by accumulating vesicles into massive intracellular clusters. ${ }^{[24]}$ In PC12 and chromaffin cells, overexpression of $\alpha \mathrm{S}$ inhibited transmitter release in a late step after docking but before fusion. ${ }^{25]}$ In vivo aggregation into oligomers directly interferes with the cell's fusion machinery. Here we address the hypothesis that aggregation of multiple $\alpha \mathrm{S}$ molecules results in the formation of nanoparticles with multivalent membrane binding sites. We show that these multivalent binding sites are able to connect opposing membranes and 


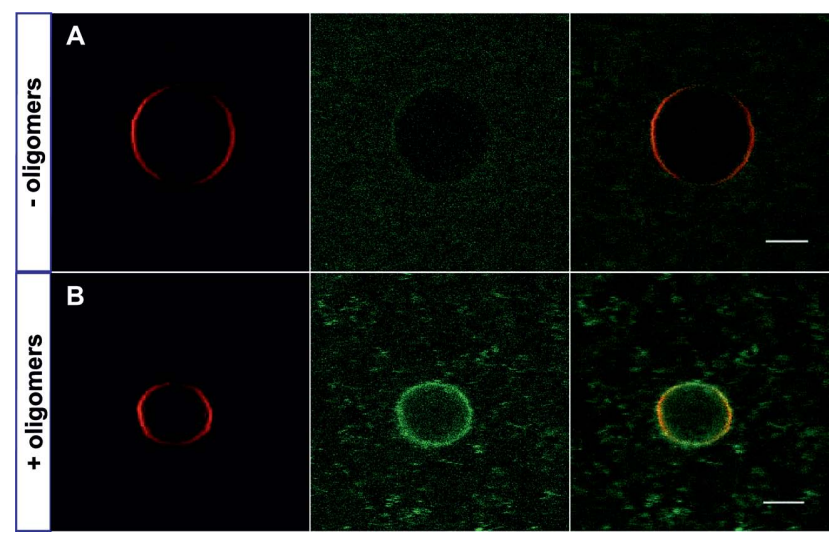

Figure 1. A) In the absence of $\alpha$ S oligomers we observe no clustering between DOPE-Rhodamine doped DOPG GUVs (red channel, first column) and NBD-PC doped DOPG LUVs (green channel, second column). The overlay between the channels (third column) confirms that there is no interaction between the vesicles. B) After the addition of oligomers we find a clear accumulation of NBD-PC labeled LUVs at the GUV surface and a clustering of LUVs in solution. The scale bars indicate $5 \mu \mathrm{m}$. The size of the LUVs is below the diffraction limit and these structures can therefore not be resolved.

result in the formation of hemifusion contacts. The toxicity of $\alpha \mathrm{S}$ oligomers may thus originate from their vesicle clustering and hemifusion docking activity, and may be generic for small, membrane-binding nanoparticles.

To study if the presence of $\alpha \mathrm{S}$ oligomers can induce the formation of membrane contacts, vesicles composed of the negatively charged phospholipid DOPG (1,2-dioleoylphosphatidylglycerol) were used as a model membrane system. Giant and large unilamellar DOPG vesicles (GUVs and LUVs) were doped with $1 \%$ fluorescently labeled lipids to be able to visualize and follow the effect of oligomers on these vesicles. Oligomeric $\alpha \mathrm{S}$ was prepared following an established protocol based on a high protein concentration and purification by size exclusion chromatography. ${ }^{[20]}$ The effect of $\alpha \mathrm{S}$ oligomers on a mixture of DOPE-Rhodamine ( $N$-(lissamine Rhodamine B sulfonyl)-1,2-dioleoyl-sn3-phosphatidylehanolamine) doped GUVs and NBD-PC (1-palmitoyl-2-\{12-[(7-nitro-2-1,3-benzoxadiazol-4-yl)amino] dodecanoyl\}-sn-glycero-3-phosphocholine) doped LUVs was visualized using confocal microscopy (Figure 1). Before the addition of oligomers to the vesicle mixture there were no signs of GUV and LUV clustering. GUVs are clearly visible as rings in the red channel of the confocal slice. The size of the LUVs is below the diffraction limit and therefore cannot be resolved. The LUVs appear as a diffuse signal in the green channel, and as expected, there is no signal originating from LUVs in the volume excluded by GUVs. The overlay between the red GUV channel and the green LUV channel confirms that there is no interaction or clustering of vesicles in the absence of $\alpha \mathrm{S}$ oligomers. Upon incubation of the vesicles with $\alpha \mathrm{S}$ oligomers, the initially diffuse LUV signal transforms into patchy structures in solution and results in what we interpret to be an accumulation of LUVs at the GUV surface.

The clustering of vesicles observed by fluorescence microscopy was confirmed by dynamic light scattering (DLS) experiments. Before adding $\alpha \mathrm{S}$ oligomers the average diameter of the LUVs was $113 \pm 6 \mathrm{~nm}$, while upon adding $\alpha \mathrm{S}$ oligomers we observed a clear increase in the average diameter to $215 \pm 8 \mathrm{~nm}$ (Figure 2B). LUV clustering also affected the width of the size distributions (DOPG alone $37 \pm 1 \mathrm{~nm}$; DOPG+oligomers $75 \pm 7 \mathrm{~nm}$ ).

The observed clustering of LUVs and GUVs suggests that oligomers are able to form connections between different membranes. Oligomers may simply connect vesicles, or induce hemifusion or even full fusion of the vesicles by merging one or both leaflets of the bilayers.

To test if the oligomer binding results in full fusion of vesicles we used a content mixing assay based on fluorescence quenching (see Figure S1A, Supporting Information, for a schematic). ${ }^{[2]}$ We prepared two batches of DOPG LUVs, one containing the fluorophore ANTS
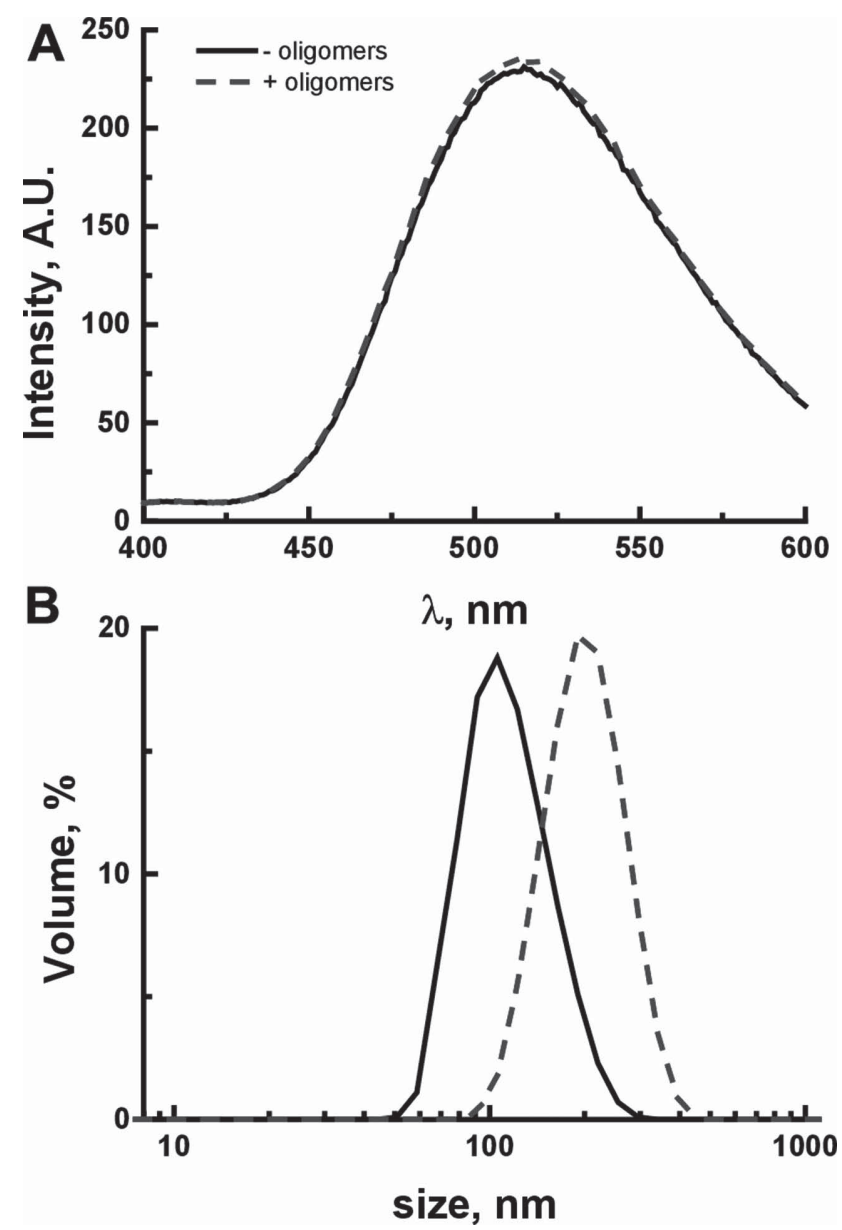

Figure 2. A) Fluorophore (ANTS) and quencher (DPX) filled DOPG vesicles were mixed in solution and the ANTS emission spectrum was recorded (black curve). $\alpha$ S oligomers were added to the vesicle containing solution and $3 \mathrm{~h}$ after addition the spectrum was recorded again (blue dashed line). The absence of a change in the emission intensity shows that the addition of oligomers did not result in content mixing and quenching of ANTS by DPX, and hence that no full fusion of the vesicles occurs. B) Dynamic light scattering measurements before (black line) and after incubation with $\alpha \mathrm{S}$ oligomers (blue dashed line) demonstrate the increase in diameter of the vesicles due to clustering from $113 \pm 6 \mathrm{~nm}$ to $215 \pm 8 \mathrm{~nm}$ (standard error based on 3 different measurements). The width of the size distribution peak also increased from $37 \pm 1 \mathrm{~nm}$ for DOPG LUVs alone to a width of $75 \pm 7 \mathrm{~nm}$ for DOPG LUVs in the presence of oligomers. 


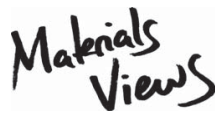

(8-aminonaphthalene-1,3,6-trisulfonicacid disodium salt), and the other containing the quencher DPX (p-xylene-bis-pyridinium bromide). Full fusion of fluorophore- and quenchercontaining vesicles will result in content mixing and hence a drop in fluorescence due to quenching of ANTS by DPX. Equal volumes of ANTS and DPX containing vesicles were mixed and incubated with $\alpha \mathrm{S}$ oligomers, while the change of fluorescence was monitored (Figure 2A). The recorded ANTS fluorescence spectra showed no drop in ANTS fluorescence upon addition of $\alpha \mathrm{S}$ oligomers and even after incubation with the vesicle mixture for $3 \mathrm{~h}$. Although our DLS data (Figure 2B) shows that oligomer binding results in vesicle clustering, oligomers cannot induce full fusion of the vesicles.

To discriminate between the formation of hemifusion contacts and $\alpha \mathrm{S}$ oligomers simply acting as connectors between vesicles, we used a Förster Resonance Energy Transfer (FRET) dilution assay that probes the mixing of membrane lipids originating from different vesicles (See Figure S1B, Supporting Information, for a schematic). The assay is based on the preparation of two populations of vesicles; one population is fluorescently labeled, while the other population is unlabeled. The membrane of the labeled vesicles contains donor and acceptor fluorophores at a concentration that ensures proximity of the fluorophores and allows for FRET, a process which is exquisitely sensitive to the distance between the fluorophores. Upon excitation of the donor fluorophore, energy can be transferred to the acceptor fluorophore. Fusion of labeled vesicles with unlabeled vesicles results in a dilution of the donor and acceptor labeled lipids by the unlabeled lipids, yielding a larger average separation between donor and acceptor. The resulting drop in FRET efficiency can easily be detected as a decrease in FRET acceptor intensity and increase in FRET donor intensity.

We prepared DOPG vesicles containing $0.8 \mathrm{~mol} \%$ of both the donor fluorophore NBD-PC and acceptor fluorophore DOPE-Rhodamine. To study any dilution of the FRET pair that may result from lipid mixing upon addition of $\alpha \mathrm{S}$ oligomers we prepared a 1:4 mixture of labeled and unlabeled vesicles, respectively. Upon addition of $\alpha \mathrm{S}$ oligomers we recorded the change in the emission spectra over time (Figure 3A).

In the absence of $\alpha \mathrm{S}$ oligomers we observe, upon excitation of the donor, a strong emission from the acceptor, DOPE-Rhodamine, and a moderate emission from the donor, which suggests effective FRET. Upon addition of oligomers the intensity of the acceptor decreases over time with a commensurate increase of the donor signal (Figure 3A). The observed changes in the emission spectra result from a decrease in the FRET efficiency caused by the dilution of the incorporated FRET-pair upon fusion of labeled and
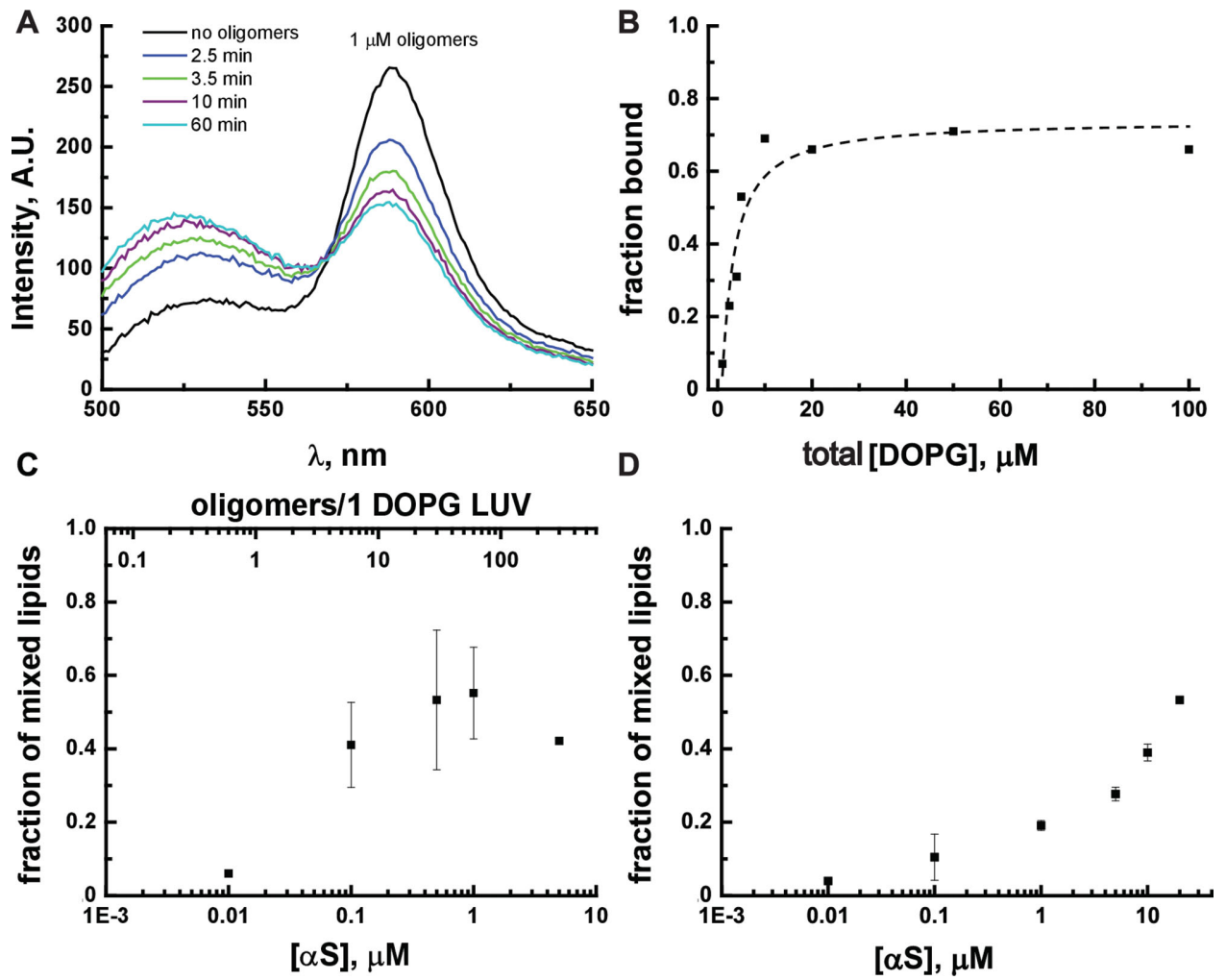

Figure 3. Membrane mixing. A) Oligomer-induced time dependent changes in the emission spectra of a NBD-PC and Rhodamine-DOPE mixture in LUVs after excitation of NBD-PC. The changes reflect a decrease in FRET efficiency due to a dilution of the FRET pair with lipids from unlabeled DOPG LUVs. B) The fraction of membrane-bound oligomers estimated from FCS experiments as a function of the lipid concentration. In the FCS experiments oligomers containing 7.5\% Alexa488 labeled $\alpha \mathrm{S}$ monomers were used. The protein concentration was kept constant. The dotted line shows the fit to the data assuming a $K_{d}$ of $1.7 \times 10^{-6} \mathrm{M}$. C) The maximal fusion efficiency expressed as the fraction of mixed lipids was determined as a function of the oligomer (monomer equivalent) concentration. Using the obtained $K_{d}$ values this concentration was expressed as a function of bound oligomers per vesicle. The fusion efficiency was limited to $\approx 50 \%$ and this maximum fusion efficiency was reached for values $<10$ oligomers / LUV. (D) Higher concentrations of monomeric $\alpha \mathrm{S}$ (compared to oligomers) are necessary to reach comparable fractions of mixed lipids. 
unlabeled vesicles. The membrane fusion-induced changes in the emission spectra upon the addition of oligomers are completed after approximately $10 \mathrm{~min}$ (Figure 3A). We repeated the experiment for different concentrations of $\alpha \mathrm{S}$ oligomers and determined the fraction of diluted lipids from the ratio between donor and acceptor fluorescence intensities after reaching a constant value for each $\alpha \mathrm{S}$ oligomer concentration (See the Supporting Information).

We observe a strong increase of the fraction of lipids mixed upon increasing the $\alpha \mathrm{S}$ concentration from $0.01 \times 10^{-6}$ to $0.1 \times 10^{-6} \mathrm{M}$ oligomers (equivalent monomer concentration). The fraction of lipids mixed reaches a plateau at $\approx 50 \%$, and increasing the $\alpha \mathrm{S}$ oligomer concentration does not result in higher values. Our data therefore indicates that only half of the labeled lipids are sensitive to the presence of oligomers.

Since oligomers have a clear effect on membrane fusion, we estimated the number of oligomers bound to each vesicle from the equilibrium dissociation constant $K_{d}$. To determine $K_{d}$ for oligomers binding to DOPG vesicles, we determined the fraction of DOPG LUV-bound $\alpha \mathrm{S}$ oligomers at different $\alpha \mathrm{S}$ oligomer concentrations using Fluorescence Correlation Spectroscopy (FCS). Membrane-bound and free (unbound) $\alpha \mathrm{S}$ oligomers were discriminated with FCS on the basis of the difference in diffusion coefficients. The fraction of membrane-bound protein, $P$ bound $=C / O_{\text {tot }}$, where $C$ is the complex concentration and $O_{\text {tot }}$ is the total protein concentration $\left(O_{\text {tot }}=O+C\right)$, was obtained as a function of the total DOPG concentration $\left(L_{\mathrm{tot}}=L+C\right.$ ) (Figure $\left.3 \mathrm{~B}\right)$. To determine the dissociation constant, $K_{d}$, the following oligomer-membrane binding equilibrium was assumed: $O+L C$ and $K_{d}=\frac{O \cdot L}{C}$, where $O$ and $L$ are the protein and lipid concentrations, respectively (for details see the Supporting Information). Rewriting the expression for $K_{d}$ in terms of the known $L_{\text {tot }}$ and $O_{\text {tot }}$ made it possible to determine $K_{d}$ (Figure $3 \mathrm{~B}$ and Figure S2, Supporting Information). By fitting the binding curve with this expression, $K_{d}$ was calculated to be $\approx 1.7 \times$ $10^{-6} \mathrm{M}$ (Figure 3B).

In the fusion experiment $40 \times 10^{-6} \mathrm{M}$ DOPG LUVs were mixed with different oligomer concentrations. Previous experiments have shown that 1 oligomer contains $\approx 30$ monomers. ${ }^{[12]}$ The DOPG vesicles have an average diameter of $113 \pm 6 \mathrm{~nm}$ (Figure 2B), and the mean headgroup area of the lipids was assumed to be $0.8 \mathrm{~nm}^{2}$. Using this information and the $K_{d}$ obtained, the protein concentrations in the fusion experiments could be expressed as the number of $\alpha \mathrm{S}$ oligomers per vesicle (Figure 3C, top axis). This figure shows that a very low average number of $\alpha \mathrm{S}$ oligomers per vesicle is sufficient to induce membrane fusion. At an average of $<10$ $\alpha \mathrm{S}$ oligomers per vesicle we observe a saturation of the fraction of mixed lipids at $\approx 50 \%$.

It has been hypothesized that $\alpha \mathrm{S}$ monomers are also able to cluster and fuse vesicles. ${ }^{[27]}$ To test this idea we used the FRET dilution assay to detect lipid mixing as a function of the concentration of $\alpha \mathrm{S}$ monomers instead of $\alpha \mathrm{S}$ oligomers. Indeed we observe the characteristic signs for lipid mixing in the assay. However, compared to oligomers, monomers were less efficient in inducing membrane fusion (Figure 3D), requiring at least 100 -fold higher monomer concentrations to induce membrane fusion compared to oligomers.
Here we have shown that oligomeric $\alpha \mathrm{S}$ has a propensity to cluster DOPG vesicles. In the presence of in vitro produced isolated oligomers, DOPG LUVs cluster and accumulate at the surface of GUVs (Figures $1 \mathrm{~B}$ and $2 \mathrm{~B}$ ). The multivalent membrane binding sites on the oligomer seem very efficient in clustering negatively charged vesicles. The effect of $\alpha \mathrm{S}$ oligomer binding is not limited to clustering; FRET experiments indicate that it also enhances the exchange of lipids between connected bilayers (Figure 3A,C). However, not all lipids in the vesicles are available for the exchange. With increasing $\alpha \mathrm{S}$ oligomer concentrations a plateau is reached in which only $50 \%-60 \%$ of the FRET-labeled lipids are diluted by unlabeled lipids (Figure 3C). This suggests that only the outer leaflets of the vesicles are affected by $\alpha \mathrm{S}$ oligomers. We attribute the slight overshoot over the expected $50 \%$ for one leaflet to the previously reported oligomer-enhanced lipid flip-flop. ${ }^{28]}$ The finding that oligomer binding does not result in content mixing of DOPG LUVs is in agreement with the formation of hemifusion contacts (Figure 2A). The fact that the fraction of membrane bound oligomers presented in Figure $3 \mathrm{~B}$ only reaches $\sim 70 \%$ and not $100 \%$ is strange. This suggests that, in spite of their well-defined aggregation number, ${ }^{[12]}$ there is a fraction of oligomers that cannot bind membranes. The membrane binding $\mathrm{N}$-terminal region is apparently not equally available in all oligomers.

Several factors may play a role in the oligomer-induced formation of hemifusion contacts between LUVs. The binding of monomeric $\alpha \mathrm{S}$ has been shown to induce a local negative Gaussian membrane curvature. ${ }^{[7]}$ Negative Gaussian curvatures $\left(1 / R_{1} R_{2}<0\right)$ are associated with saddle surfaces and hence the formation of fusion intermediates. ${ }^{[29]}$ Since the membrane binding properties are largely preserved in the oligomer, oligomers may also favor the formation of saddle surfaces. Moreover the close contact between opposing membranes that is established by binding of oligomers to multiple vesicles may in itself be sufficient to drive the formation of hemifusion. The release of hydration energy when contacts with an intermembrane distance of $\leq 1 \mathrm{~nm}$ are formed can be enough to compensate for the elastic energy of hemifusion stalks. ${ }^{[29]}$ Cationic polymers are known to induce aggregation of anionic vesicles. When the polymer layer is thin, this aggregation is followed by full membrane fusion, but when the adsorbed cationic polymer layer is too thick, it forms a barrier that prevents fusion. ${ }^{[30]}$ A low resolution oligomer structure obtained by small angle X-ray scattering shows that oligomers are elongated structures with a long axis of $\approx 15-18 \mathrm{~nm}$ and a diameter of $4.5-10 \mathrm{~nm} \cdot{ }^{[14,31]}$ Considering the required small inter-membrane distance this suggests that to induce hemifusion oligomers will have to partially insert into the bilayer and perhaps deform upon binding.

By obtaining $K_{d}$ for the oligomer-membrane binding reaction (Figure $3 \mathrm{~B}$ ) we were able to estimate the mean number of bound oligomers required for hemifusion (Figure 3C). The fusion plateau is reached at fewer than $\approx 10$ oligomers per vesicle indicating that the formation of hemifusion contacts is very efficient. This is in good agreement with observations for fusogenic proteins and peptides. ${ }^{[32]}$ The energy landscape of the fusion reaction is relatively flat and fusion itself is therefore not a very specific 
reaction. ${ }^{[33]}$ The complex fusion machinery employed by cells has been suggested to be involved in controlling docking rather than in carrying out fusion itself. ${ }^{[33]}$ The toxicity of oligomers may thus result from the spontaneous formation of hemifusion contacts between membranes and subsequent interference with the fusion control machinery.

In our experiments with oligomers, fusion did not proceed beyond the hemifusion state. The transformation of a hemifusion contact to an expanding pore is more energy demanding and probably controlled by the buildup of lateral membrane tension. ${ }^{29,34]}$ Binding or incorporation of oligomers is however known to result in a (transient) increase in membrane permeability. ${ }^{[20,28]}$ The buildup of membrane tension by bound proteins necessary for full fusion is therefore probably not possible in this model system. For other fusogenic peptides, additional destabilization by the addition of $\mathrm{Ca}^{2+}$ ions is required to achieve full fusion. ${ }^{[35]}$

Because of the presence of multiple membrane binding domains $\alpha \mathrm{S}$ oligomers are very efficient in connecting DOPG vesicles, but vesicles also fuse in the presence of monomeric protein albeit at higher concentrations (Figure 3D). The observation that monomers can induce fusion does not agree with the inhibition of vesicle fusion by $\alpha \mathrm{S}$ observed by Kamp et al. ${ }^{[36]}$ Interestingly the formation of hemifusion contacts in the presence of $\alpha \mathrm{S}$ monomers becomes very efficient around the critical aggregation concentration. ${ }^{[37]}$ This suggests that also in this experiment performed with monomeric $\alpha \mathrm{S}$, incipient multimeric $\alpha \mathrm{S}$ may be responsible for hemifusion.

By disrupting the integrity of cellular membranes, oligomers are thought to decrease cell viability. This toxic mechanism is currently under debate; although oligomeric $\alpha \mathrm{S}$ has been shown to disrupt the integrity of anionic phospholipid vesicles, bilayers with more physiological relevant lipid compositions are hardly affected. ${ }^{[21]}$ Our data suggest that there is an additional mechanism involved in the toxicity of $\alpha \mathrm{S}$ oligomers. The protein $\alpha \mathrm{S}$ binds membranes by adopting an amphipathic $\alpha$-helical structure. The ability to adopt a membrane-binding $\alpha$-helical conformation is largely preserved in oligomeric $\alpha \mathrm{S} .{ }^{[20,21]}$ The membrane-bound oligomers may interfere with the physiological function of the $\alpha \mathrm{S}$ protein. Ultrastructural analysis shows that with increasing cellular $\alpha \mathrm{S}$ levels transport vesicles bud normally from their parent membrane, but at the cell periphery these now heterogeneously sized vesicles no longer dock and fuse normally. At the plasma membrane morphologically undocked vesicles appear, and with increasing $\alpha \mathrm{S}$ levels massive intracellular vesicular clusters show up. ${ }^{[2]}$ Increased cellular $\alpha$ S concentrations have been associated with $\alpha \mathrm{S}$ aggregation and the formation of toxic $\alpha \mathrm{S}$ oligomers. The oligomer-induced formation of hemifusion contacts between DOPG vesicles indicates that the vesicle docking and fusion problems observed in vivo may directly result from the presence of oligomers. While the oligomers we study here have a very specific structure and aggregation number in vitro, it is reasonable to assume that oligomers found in vivo may exhibit similar properties because of their multivalent nature.

As proposed for $\alpha \mathrm{S}$ oligomers, the toxicity of nanoparticles has been suggested to involve nanoscale hole formation or membrane thinning. Indeed cationic gold, polystyrene, and core-shell nanoparticles are able to bind and disrupt negatively charged vesicles, ${ }^{[38]}$ but membrane disruption depends on the number of charges on the surface and probably also on the membrane charge density. ${ }^{[39]}$ Interestingly nanoparticles with a diameter smaller than tens of $\mathrm{nm}$ have been reported to be more toxic than larger nanoparticles. These observations suggest that the toxic mechanism we propose here may not be limited to the specific $\alpha \mathrm{S}$ oligomers (aggregation number of $\approx 30$ ) studied. When small cationic nanoparticles become available in the cell cytoplasm, binding to multiple vesicles or other membrane structures may result in the close membrane contacts required for (hemi)fusion. Therefore the clustering and possible fusion of membrane bound cellular structures may also contribute to the toxicity of small cationic nanoparticles.

\section{Supporting Information}

Supporting Information is available from the Wiley Online Library or from the author.

\section{Acknowledgements}

This work was financially supported by the "Nederlandse Organisatie voor Wetenschappelijk Onderzoek" (NWO) through the NWO-CW TOP Program No. 700.58.302 to V.S. Additional funding was provided by Stichting International Parkinson Fonds. We thank Kirsten van Leijenhorst-Groener and Nathalie Schilderink for assistance in expression and purification of alpha-synuclein. We thank Niels Zijlstra for help with performing the FCS experiments and data analysis. V.S. also acknowledges support from the "Stichting voor Fundamenteel Onderzoek der Materie (FOM)" through the FOM program "A Single Molecule View on Protein Aggregation."

[1] A. Iwai, E. Masliah, M. Yoshimoto, N. Ge, L. Flanagan, H. A. de Silva, A. Kittel, T. Saitoh, Neuron 1995, 14, 467.

[2] E. Rhoades, T. F. Ramlall, W. W. Webb, D. Eliezer, Biophys. J. 2006, 90, 4692.

[3] a) W. S. Davidson, A. Jonas, D. F. Clayton, J. M. George, J. Biol. Chem. 1998, 273, 9443; b) M. Stockl, P. Fischer, E. Wanker, A. Herrmann, J. Mol. Biol. 2008, 375, 1394.

[4] C. Guardia-Laguarta, E. Area-Gomez, C. Rub, Y. Liu, J. Magrane, D. Becker, W. Voos, E. A. Schon, S. Przedborski, J. Neurosci. 2014, 34, 249.

[5] Y.-J. Chai, D. Kim, J. Park, H. Zhao, S.-J. Lee, S. Chang, FEBS Lett. 2013, 587, 452.

[6] S. Bellani, V. L. Sousa, G. Ronzitti, F. Valtorta, J. Meldolesi, E. Chieregatti, Commun. Integr. Biol. 2010, 3, 106.

[7] A. R. Braun, E. Sevcsik, P. Chin, E. Rhoades, S. Tristram-Nagle, J. N. Sachs, J. Am. Chem. Soc. 2012, 134, 2613.

[8] A. Leftin, C. Job, K. Beyer, M. F. Brown, J. Mol. Biol. 2013, 425, 2973.

[9] I. Dikiy, D. Eliezer, Biochim. Biophys. Acta 2012, 1818, 1013.

[10] a) J. Burre, M. Sharma, T. Tsetsenis, V. Buchman, M. R. Etherton, T. C. Sudhof, Science 2010, 329, 1663; b) D. C. DeWitt, E. Rhoades, Biochemistry 2013, 52, 2385.

[11] a) R. Kayed, E. Head, J. L. Thompson, T. M. Mclntire, S. C. Milton, C. W. Cotman, C. G. Glabe, Science 2003, 300, 486; 
b) K. M. Danzer, W. P. Ruf, P. Putcha, D. Joyner, T. Hashimoto, C. Glabe, B. T. Hyman, P. J. McLean, FASEB J. 2011, 25, 326; c) R. Cappai, S. L. Leck, D. J. Tew, N. A. Williamson, D. P. Smith, D. Galatis, R. A. Sharples, C. C. Curtain, F. E. Ali, R. A. Cherny, J. G. Culvenor, S. P. Bottomley, C. L. Masters, K. J. Barnham, A. F. Hill, FASEB J. 2005, 19, 1377.

[12] N. Zijlstra, C. Blum, I. M. Segers-Nolten, M. M. Claessens, V. Subramaniam, Angew Chem. Int. Ed. Engl. 2012, 51, 8821.

[13] N. Zijlstra, M. A. E. Mireille Claessens, C. Blum, V. Subramaniam, Biophys. J. 2014, 106, 440.

[14] N. Lorenzen, S. B. Nielsen, A. K. Buell, J. D. Kaspersen, P. Arosio, B. S. Vad, W. Paslawski, G. Christiansen, Z. Valnickova-Hansen, M. Andreasen, J. J. Enghild, J. S. Pedersen, C. M. Dobson, T. P. Knowles, D. E. Otzen, J. Am. Chem. Soc. 2014, 136, 3859.

[15] a) M. Schulz, A. Olubummo, W. H. Binder, Soft Matter 2012, 8, 4849; b) A. Verma, F. Stellacci, Small 2010, 6, 12.

[16] a) C. M. Goodman, C. D. McCusker, T. Yilmaz, V. M. Rotello, Bioconjugate Chem. 2004, 15, 897; b) J. Q. Lin, H. W. Zhang, Z. Chen, Y. G. Zheng, ACS Nano 2010, 4, 5421.

[17] a) E. Frohlich, Int. J. Nanomedicine 2012, 7, 5577; b) P. K. Auluck, G. Caraveo, S. Lindquist, Annu. Rev. Cell Dev. Biol. 2010, 26, 211.

[18] P. R. Leroueil, S. A. Berry, K. Duthie, G. Han, V. M. Rotello, D. Q. McNerny, J. R. Baker Jr., B. G. Orr, M. M. Holl, Nano Lett. 2008, 8, 420.

[19] M. Volles, P. Lansbury, Biochemistry 2002, 41, 4595.

[20] B. D. van Rooijen, M. M. Claessens, V. Subramaniam, Biochim. Biophys. Acta 2009, 1788, 1271.

[21] A. N. Stefanovic, M. T. Stockl, M. M. Claessens, V. Subramaniam, FEBS J. 2014, 281, 2838.

[22] E. Sparr, M. F. Engel, D. V. Sakharov, M. Sprong, J. Jacobs, B. de Kruijff, J. W. Hoppener, J. A. Killian, FEBS Lett. 2004, 577, 117.

[23] H. A. Lashuel, B. M. Petre, J. Wall, M. Simon, R. J. Nowak, T. Walz, P. T. Lansbury, J. Mol. Biol. 2002, 322, 1089.

[24] A. Gitler, B. Bevis, J. Shorter, K. Strathearn, S. Hamamichi, L. Su, K. Caldwell, G. Caldwell, J. Rochet, J. McCaffery, C. Barlowe, S. Lindquist, Proc. Natl. Acad. Sci. USA 2008, 105, 145.

[25] K. E. Larsen, Y. Schmitz, M. D. Troyer, E. Mosharov, P. Dietrich, A. Z. Quazi, M. Savalle, V. Nemani, F. A. Chaudhry, R. H. Edwards, L. Stefanis, D. Sulzer, J. Neurosci. 2006, 26, 11915.
[26] H. Ellens, J. Bentz, F. C. Szoka, Biochemistry 1985, 24, 3099.

[27] J. Diao, J. Burré, S. Vivona, D. J. Cipriano, M. Sharma, M. Kyoung, T. C. Südhof, A. T. Brunger, eLife 2013, 2.

[28] M. Stockl, M. M. Claessens, V. Subramaniam, Mol. Biosyst. 2012, $8,338$.

[29] L. Chernomordik, M. M. Kozlov, J. Zimmerberg, J. Membr. Biol. 1995, 146, 1.

[30] A. A. Yaroslavov, A. V. Sybachin, E. Kesselman, J. Schmidt, Y. Talmon, S. A. Rizvi, F. M. Menger, J. Am. Chem. Soc. 2011, 133, 2881.

[31] L. Giehm, D. I. Svergun, D. E. Otzen, B. Vestergaard, Proc. Natl. Acad. Sci. USA 2011, 108, 3246.

[32] a) G. van den Bogaart, M. G. Holt, G. Bunt, D. Riedel, F. S. Wouters, R. Jahn, Nat. Struct. Mol. Biol. 2010, 17, 358; b) E. M. Reuven, Y. Dadon, M. Viard, N. Manukovsky, R. Blumenthal, Y. Shai, Biochemistry 2012, 51, 2867.

[33] R. Jahn, H. Grubmüller, Curr. Opin. Cell Biol. 2002, 14, 488.

[34] L. Chernomordik, A. Chanturiya, J. Green, J. Zimmerberg, Biophys. J. 1995, 69, 922.

[35] a) K. Hu, J. Carroll, S. Fedorovich, C. Rickman, A. Sukhodub, B. Davletov, Nature 2002, 415, 646; b) B. P. Jena, Protein Pept. Lett. 2009, 16, 712; c) S. S. Vogel, J. Zimmerberg, Proc. Natl. Acad. Sci. USA 1992, 89, 4749.

[36] F. Kamp, N. Exner, A. K. Lutz, N. Wender, J. Hegermann, B. Brunner, B. Nuscher, T. Bartels, A. Giese, K. Beyer, S. Eimer, K. F. Winklhofer, C. Haass, EMBO J. 2010, 29, 3571.

[37] M. E. van Raaij, I. M. Segers-Nolten, V. Subramaniam, Biophys. J. 2006, 91, L96.

[38] M. Laurencin, T. Georgelin, B. Malezieux, J. M. Siaugue, C. Menager, Langmuir 2010, 26, 16025.

[39] R. R. Arvizo, O. R. Miranda, M. A. Thompson, C. M. Pabelick, R. Bhattacharya, J. D. Robertson, V. M. Rotello, Y. S. Prakash, P. Mukherjee, Nano Lett. 2010, 10, 2543.

Received: September 4, 2014 Revised: November 16, 2014

Published online: January 15, 2015 